\title{
A Case Study in Model-driven Synthetic Biology
}

\author{
David Gilbert, Monika Heiner, Susan Rosser, Rachael Fulton, Xu Gu and Maciej \\ Trybiło
}

\begin{abstract}
We report on a case study in synthetic biology, demonstrating the modeldriven design of a self-powering electrochemical biosensor. An essential result of the design process is a general template of a biosensor, which can be instantiated to be adapted to specific pollutants. This template represents a gene expression network extended by metabolic activity. We illustrate the model-based analysis of this template using qualitative, stochastic and continuous Petri nets and related analysis techniques, contributing to a reliable and robust design.
\end{abstract}

\section{Motivation}

One of the greatest challenges in modern bioscience is arguably the development of techniques for the engineering of living systems in a rigorous manner. This is the domain of the emerging discipline of "Synthetic Biology" [HP06], which can be defined as the design and construction of new biological parts, devices, and systems, as well as the re-design of existing natural biological systems for useful purposes [Syn08]. One aspect of Synthetic Biology which distinguishes it from conventional genetic engineering is a heavy emphasis on the development of foundational technologies that make the engineering of biology easier and more reliable.

David Gilbert, Rachael Fulton, Xu Gu, Maciej Trybiło

Bioinformatics Research Centre, University of Glasgow, Glasgow G12 8QQ, Scotland, UK, e-mail: drg@brc.dcs.gla.ac.uk,0307842f@student.gla.ac.uk,gux@brc.dcs.gla.ac.uk, trybilom@dcs.gla.ac.uk

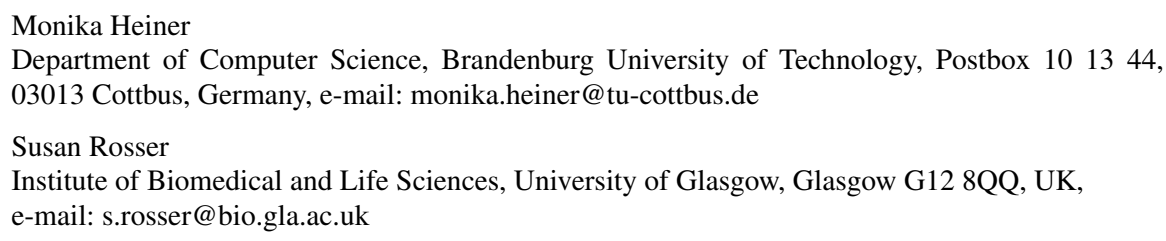


We report on a case study in synthetic biology [Gla07], demonstrating the modeldriven construction of a completely novel type of self-powering electrochemical biosensor, called ElectrEcoBlu. The novelty lies in the fact that the response signal is an electrochemical mediator which enables electrical current to be generated in a microbial fuel cell. ElectrEcoBlu functions as a biosensor for a range of important and widespread environmental organic pollutants which stimulate the biosensor to produce its own electrical power output. The system has the potential to be used for self-powered long term in situ and online monitoring with an electrical readout.

Our approach exploits a range of state-of-the art modelling techniques [GHL07] to guide the design and construction of this novel synthetic biological system in order to ensure that its behaviour is reliable and robust under a variety of conditions. This was facilitated by the entire team - molecular biologists and engineers/modellers - working in an integrated laboratory environment, using Petri nets as a communication means and following an iterative construction process as given in Fig. 1. An essential result of the design process is a general template of a biosensor, which can be instantiated to be adapted to special pollutants. This template represents a gene expression network extended by metabolic activity. We demonstrate the model-based analysis of this template, and by this way of the design of the biosensor, using qualitative, stochastic and continuous Petri nets and related analysis techniques.

Fig. 1 Model-driven synthetic biology. Computer modelling and analysis guides the design and construction in order to ensure behaviour, which is reliable and robust under a variety of conditions.

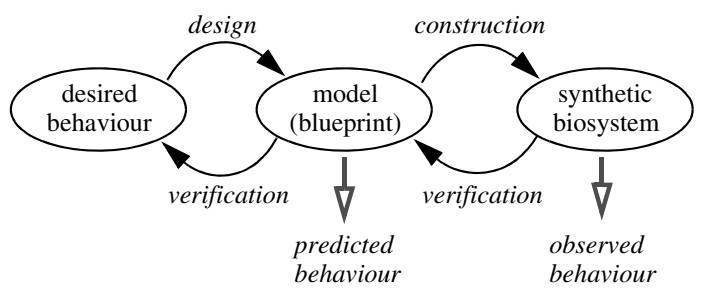

\section{Biochemical Context}

Public concern and legislation are demanding better environmental control and monitoring of pollutants. Biosensors are being developed in the fields of environment, bioprocess control, food, agriculture, military, and medical industries. Biosensor sensitivity and selectivity depend essentially on the properties of the biorecognition elements to be used for analyte binding.

The discovery of transcriptional activators and their corresponding promoter sequences has made possible the development of bacterial biosensors for pollutants. Modified cell biosensors are constructed by fusing a reporter gene (an enzyme or a fluorescent protein e.g. GFP) to a promoter element that is induced by the presence of a target compound. In the presence of an organic contaminant the transcriptional 
activator changes its three dimensional structure, becoming operative, and transcription of the reporter gene is enhanced. The gene is transcribed to form mRNA which is then translated into a protein which performs the biochemical activity (in the case of enzymes) or fluoresces (e.g. GFP). The resulting increase in reporter gene product is then detected by measuring the activity of the reporter enzyme or the fluorescence of the reporter protein. Thus, under appropriate conditions, a direct correlation between contaminant concentration and reporter product can be established.

One holy grail of environmental biosensors is to create a system which can be left in the field continuously monitoring and remotely sending electronic signals back to a computer. One major problem is how to power such a device so that frequent expensive battery changes are not necessary. One possible source of renewable energy for powering biosensor devices are microbial fuel cells (MFC) in which microorganisms oxidize compounds such as glucose, acetate or wastewater. The electrons gained from this oxidation are transferred to an electrode. In the past, external, expensive, soluble redox mediators have consistently been added to MFCs to enhance electron transfer. Pseudomonas aeruginosa has been shown to produce its own electron transporters, pyocyanin (PYO), which can function as electron-carrying redox mediators increasing electrical power output of MFCs.

Our project aimed to use a synthetic biology approach to combine the production of an environmental biosensor for economically important industrial environmental pollutants with a microbial fuel cell which can produce its own electricity. The intention is that the cells will recognise the presence of a pollutant via a modular interchangeable range of pollutant-specific transcriptional activator proteins and enhance electricity generation in a microbial fuel cell by inducing genes for the synthesis of the electron mediator PYO which function as novel reporter genes.

The recognition element of the designed biosensor system is a pollutant responsive transcriptional activator XylR (DntR) which binds the important environmental pollutant toluene (salicylate). The reporter element of the biosensor consists of the enzymes $S$-adenosylmethionine-dependent $N$-methyltransferase (PhzM) and flavindependent hydroxylase (PhzS) which convert the precursor compound phenazine-1carboxamide (PCA) to PYO in the biosynthetic pathway cloned from Pseudomonas aeruginosa into E. coli and a non-pathogenic Pseudomonad strain.

The molecular biologists of our team constructed an initial diagram to describe the system, using a fairly informal graphical syntax, see Figure 2. The generic form of the transcription factor ('tf' for the gene, and ' $\mathrm{TF}$ ' for the protein product) represents both XylR (toluene detecting) and DntR (salicylate detecting). In outline, essential steps that we used to develop and refine our model are:

1. Simplification by abstracting away the mRNA, thus combining transcription and translation.

2. Summarizing pollutant-specific transcriptional activator proteins under the term TF.

3. Combining the PhzM and PhzS components to give one step from PCA to PYO.

4. Developing a variant of the model with a positive feedback loop (pfb). 
By doing so, we obtain a gene expression network, extended by metabolic activity, i.e. the model combines different abstraction levels: gene activity as well as metabolic activity, in the style of $\left[\mathrm{vHNM}^{+} 00\right]$. This represents deliberately a minimal model concentrating on the most essential facts necessary to investigate the system's signal/response behaviour. To be able to analyse the system before having constructed it, we are going to apply formal modelling techniques allowing the computer-based evaluation of the system under construction.

Fig. 2 The general biosensor scheme in two versions: without/with the positive feedback (pfb). Thick arrows represent protein coding genes, thin right-angle arrows represent promotors optionally labelled with the transcription factor, and thin straight arrows represent biochemical reactions. Note that the first instance of the 'tf' protein coding gene is constitutively expressed.

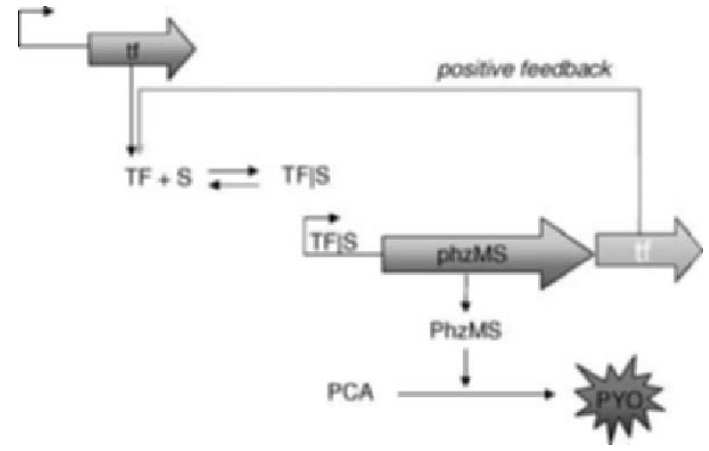

\section{Framework}

We have used a framework [GHL07] which integrates the qualitative, stochastic and continuous paradigms, as a basis for our overall approach to modelling and analysing the biosynthetic pathways, compare Fig. 3. Each perspective adds its contribution to the understanding of the system, thus the three approaches do not compete, but complement each other.

In summary, the qualitative time-free description is the most basic one, with discrete values representing numbers of molecules or levels of concentrations. The qualitative description abstracts over two timed, quantitative models. In the stochastic description, discrete values for the amounts of species are retained, but a stochastic rate is associated with each reaction. The continuous description models amounts of species using continuous values and associates a deterministic rate with each reaction, which now occurs continuously. These two time-dependent models can be mutually approximated by propensity (hazard) functions belonging to the stochastic world; see [GHL07] for more details.

This framework can be applied to a variety of formalisms; we specify stochastic models by stochastic Petri nets defining reaction rate equations (RREs), and continuous models by continuous Petri nets defining ordinary differential equations (ODEs). In the following we assume basic knowledge in the standard Petri net terminology; see e.g. [BK02, DA05, $\mathrm{MBC}^{+}$95] for introduction and related definitions. 


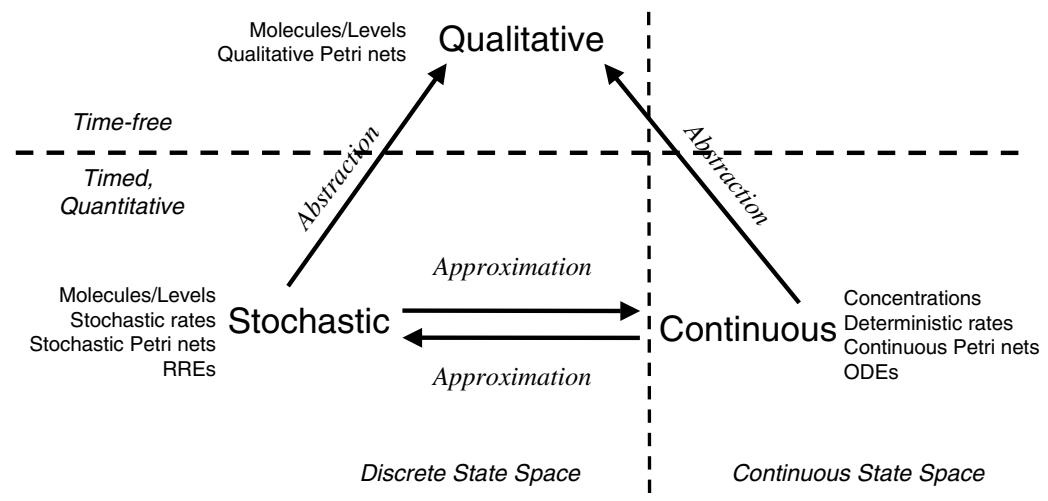

Fig. 3 Conceptual framework of our computational methods.

\section{Qualitative Approach}

Modelling From the graphical representation of the system given in Figure 2 and its accompanying explanations we derive a qualitative Petri net describing a general kernel biosensor template, and also one possible extension with pfb, see Figure 4. This template and the pfb variant may be instantiated according to Table 1, creating dedicated biosensors for different pollutants. Other variants are possible, including switches and clamps, which we do not discuss here for lack of space.

The Petri net represents an extended gene expression network comprising transitions of various abstraction levels:

- gene expression: TF expression, reporter expression;

- association/deassociation: TFS association, TFS deassociation;

- enzymatic reaction: response production;

- degradation: TF/TFS/reporter/response degradation.

The transition $T F$ expression is an input transition (transition without preplaces), modelling a constitutively expressed transcription factor, i.e. a gene which is constantly active. The degradation transitions are output transitions (transitions without postplaces). They model the fact that species naturally degrade, i.e. their concentration diminishes if they are not produced continuously.

The two essential components of a biosensor are easily identified: the recognition element (upper part), and the reporter element (lower part), both coupled by the TFS complex. In the recognition element, the signal (pollutant) forms a complex (TFS) with a constitutively expressed transcription factor (TF). The TFS complex may accelerate the TF expression, thus facilitating faster TFS association; in this case we get a pfb. In the reporter element, the two read arcs (having a black dot as arrow head) reflect the signalling cascade: TFS $\rightarrow$ reporter $\rightarrow$ response. In the following we analyse the template, and by this way all its instances. 

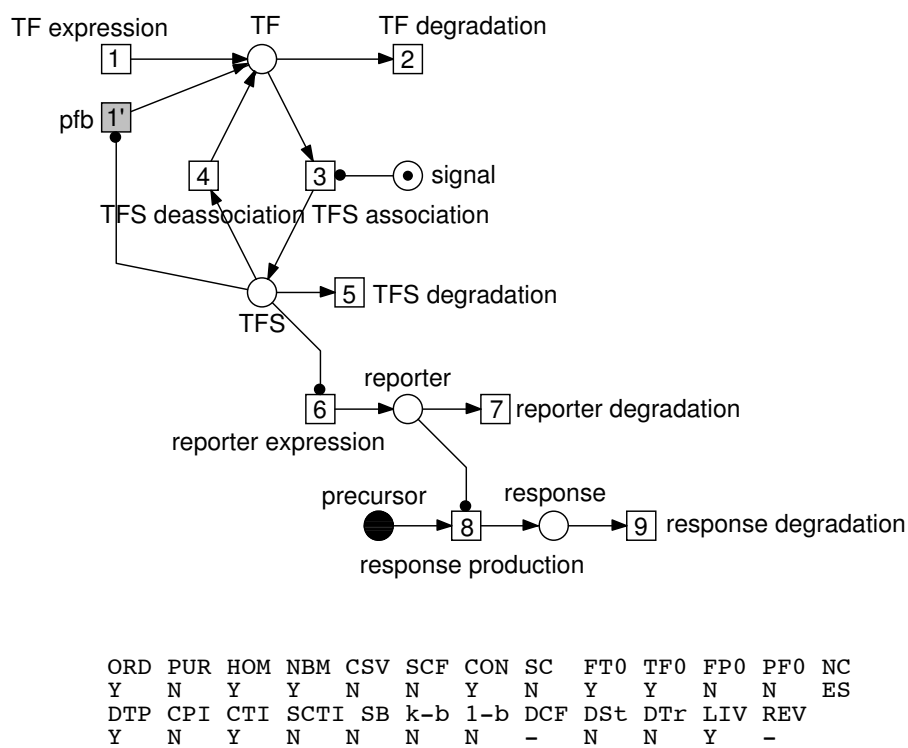

Fig. 4 Qualitative Petri net(s) of the biosensor template. The kernel system is given with white transitions, and the positive feedback (pfb) variant has an additional transition indicated in grey. The place given in black models the precursor, which is assumed to be available in sufficiently large amounts; hence it is neglected in the model analyses. The two-lines result vector given at the bottom summarizes the main qualitative analysis results; see [HGGH07] for more details.

Table 1 Possible instantiations for the places in the biosensor template.

\begin{tabular}{ll}
\hline place & instances \\
\hline signal & toluene, salicylate \\
TF & $X y l R, D n t R$ \\
TFS & $X y l R \mid S$, Dnt $R \mid S$ \\
reporter & $P h z M S$ as combination of $P h z M$ and $P h z S$ \\
precursor & phenazine-1-carboxamide $(P C A)$ \\
response & pyocyanin $(P Y O)$ \\
\hline
\end{tabular}

Analysis Having established initial confidence in the model behaviour by playing the token game, both systems were formally analysed. We list here the most essential analysis results only. For a summary see the two-lines result vector given at the bottom of Figure 4, for more explanatory details see [HGGH07].

The Petri net has input transitions and output transitions, i.e. it is an open system. Input transitions are always enabled, therefore they are able to fire arbitrarily often, making the Petri net unbounded. Consequently, the Petri net is not covered by P-invariants (CPI). Actually, there is only one minimal P-invariant, which comprises merely the place signal. That means that the token number on this place never changes under any firing, reflecting the model assumption that the signal (pollutant) 
is constantly there at a strength as chosen by the initial marking. Therefore, this place requires at least one token in the initial marking to allow its posttransition to fire. On the contrary, all other places - empty in the initial marking - are unbounded, i.e. the token number may rise to infinity if we consider the model under any timing behaviour. The proof of boundedness under given timing constraints, as e.g. by determining a steady state, is left to the quantitative analyses, see Section 5.

Having the initial marking, we consider liveness. The Petri net is ordinary, i.e. all arc weights are equal to 1, and the net belongs to the structural net class Extended Simple (ES). The Petri net with the given initial marking has the deadlock trap property (DTP). The DTP involves liveness for ordinary ES nets. Because the net is live, there are no dead transitions and no dead states. That basically means that all reactions will take place forever. Because the net is ES, the liveness is guaranteed for any timing constraints.

We compute the T-invariants to get the subprocesses, from which the whole infinite system behaviour is comprised. The Petri net without pfb is covered by the following minimal T-invariants (CTI), all enjoying an obvious biological meaning:

$$
\begin{aligned}
& y_{1}=\{\text { TF expression, } T F \text { degradation }\}, \\
& y_{2}=\{\text { TF expression, } T F S \text { association, TFS degradation }\}, \\
& y_{3}=\{\text { TF S association, } T \text { F S deassociation }\}, \\
& y_{4}=\{\text { reporter expression, reporter degradation }\}, \\
& y_{5}=\{\text { response production, response degradation }\} .
\end{aligned}
$$

The Petri net with pfb has additionally the following two T-invariants (the counterparts to $y_{1}, y_{2}$, replacing $T F$ expression by $p f b$ ):

$$
\begin{aligned}
& y_{6}=\{p f b, T F \text { degradation }\}, \\
& y_{7}=\{p f b, T F S \text { association, } T F S \text { degradation }\} .
\end{aligned}
$$

One of the benefits of using the qualitative approach at this early stage of system design was that the systems could be modelled and analysed without any quantitative parameters. Moreover the qualitative step helps in identifying suitable initial markings and potential quantitative analysis techniques.

\section{Quantitative Approaches}

Modelling To transform the validated qualitative Petri net into quantitative ones, we need to assign to all reactions their rate functions, which generally employ the current state of the reactions' substrates, or - in Petri net terms - the current marking of the transitions' preplaces. Table 2 gives for each reaction (transition): the reaction equation, the rate function and the involved rate constant(s). The rate functions are used in the stochastic model as the propensity (hazard) functions, determining the current stochastic firing rates, and in the continuous model as the deterministic rate functions, determining the current deterministic firing rates. The conversion of stochastic and deterministic rate constants into each other is well understood, see e.g. [Wil06], especially it holds that they are equivalent for first-order reactions. 
Table 2 The reaction equation, rate function, and rate constant(s) for each reaction (transition ${ }^{a}$ ). The running numbers correspond to the transition numbers in Figure 4. For better readability we use the abbreviations of the instances employed, compare Table 1, and $s$ for the signal. For the concrete values or value ranges of the rate constants see [HGGH07].

\begin{tabular}{|c|c|c|c|c|}
\hline \# & reaction & equation & rate function ${ }^{b}$ & rate constant \\
\hline 1 & $\phi$ & $\rightarrow \quad T F$ & $c_{1}$ & $c_{1}=\alpha_{T F}$ \\
\hline $1^{\prime}, \mathrm{pfb}$ & $\phi$ & $\rightarrow \quad T F$ & $\left(c_{11} \cdot T F S\right) /\left(c_{12}+T F S\right)$ & $c_{11}=\beta_{T F}, c_{12}=\gamma_{T F}$ \\
\hline 2 & $T F$ & $\rightarrow \phi$ & $c_{2} \cdot T F$ & $c_{2}=\delta_{T F}$ \\
\hline 3 & $T F+s$ & $\rightarrow \quad T F S$ & $c_{3} \cdot s \cdot T F$ & $c_{3}=\beta_{T F S}$ \\
\hline 4 & $T F S$ & $\rightarrow \quad F T$ & $c_{4} \cdot T F S$ & $c_{4}=k_{d}$ \\
\hline 5 & $T F S$ & $\rightarrow \phi$ & $c_{5} \cdot T F S$ & $c_{5}=\delta_{T F S}$ \\
\hline 6 & $\phi$ & PhzMS & $\left(c_{61} \cdot T F S\right) /\left(c_{62}+T F S\right)$ & $c_{61}=\beta_{P h z M S}, c_{62}=\gamma_{P h z M S}$ \\
\hline 7 & $P h z M S$ & $\rightarrow \phi$ & $c_{7} \cdot P h z M S$ & $c_{7}=\delta_{P h z M S}$ \\
\hline 8 & $\phi$ & $\rightarrow \quad P Y O$ & $c_{8} \cdot P h z M S$ & $c_{8}=\alpha_{P Y O}$ \\
\hline 9 & $P Y O$ & $\rightarrow \phi$ & $c_{9} \cdot P Y O$ & $c_{9}=\delta_{P Y O}$ \\
\hline
\end{tabular}

${ }^{a}$ The preplaces of a transition correspond to the reaction's substrates, and its postplaces to the reaction's products.

${ }^{b}$ Reactions 1', 6 employ Michaelis-Menten kinetics, while all others follow the mass action kinetics.

Finding the rate constants proved to be a difficult and time consuming process. It involved both searches for scientific papers and also discussions with the biologists on our team. Sometimes the exact value for a parameter could not be found due to lack of published material on the reactions involved, however the biologists managed to identify a suitable range of values between which the parameter would fall. The values for the TF were estimated using average values for bacterial transcription factors, those for PhzMS using standard rates for similar proteins, and those for $\mathrm{PYO}$ using rates from the literature $\left[\mathrm{OAM}^{+} 03, \mathrm{PGS}^{+} 07\right]$.

In the following we illustrate the strength of quantitative approaches by selected examples with special emphasis on sensitivity analysis, which aims at the identification of those parameters to which a system is sensitive; i.e. small changes in a parameter's value significantly affect the system behaviour. We start with the stochastic approach to exclude eccentric system behaviour caused by stochastic noise, before considering the averaged behaviour in the deterministic continuous approach.

Stochastic analysis The class of stochastic Petri nets [BK02, $\left.\mathrm{MBC}^{+} 95\right]$ associates an exponentially distributed firing rate (waiting time) with each transition, specified by a firing rate $\lambda$. Generally, this state-dependent firing rate is defined by a propensity (hazard) function. Table 2 provides the details which permit reading the net in Figure 4 as a stochastic Petri net, specifying at the same time RREs.

The unboundedness of the underlying qualitative model precludes the use of all standard Markov analysis techniques, which are based on the state transition matrix. Applying Gillespie's exact simulation algorithm [Gil77] produces data describing the dynamic evolution of the biological system over time. Note that the template describes the model for one cell; the organism that we use as the 'chassis' for our synthetic system is E. Coli, which is unicellular. However the bacteria exist in colonies 
comprising many cells, each of which contributes to the total production of the response. Thus we have carried out simulations for different size colonies in order to investigate the effect on the observed behaviour, under the reasonable assumption that there is no interaction between individuals in the colony. Figure 5 shows the output of the response (PYO) over time for a signal $s=10 \mu \mathrm{M}$. Each graph represents a different number of cells being simulated (1, 10, 100 and 1000), averaged over 10 runs. The noise decreases as the number of cells increases, thus behaviour of the stochastic model approaches that of the deterministic model (see Figure 7). Moreover it is obvious that the system reaches a steady state in all shown cases, determining the value at which the response saturates.

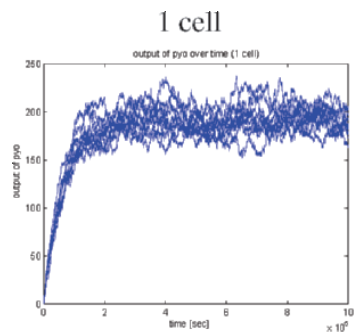

100 cells

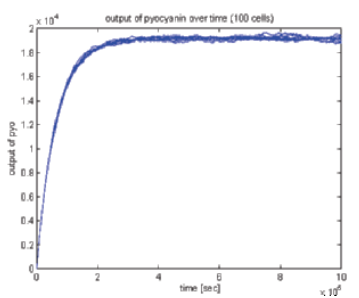

10 cells

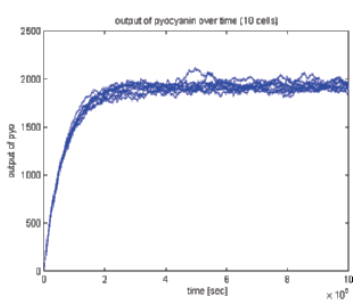

1000 cells

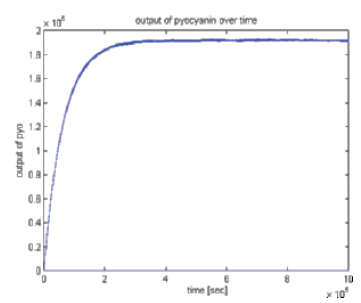

Fig. 5 Diagrams displaying the response (PYO) in number of molecules to a signal $s=10 \mu \mathrm{M}$ over time produced by simulations for $1,10,100$ and 1000 cells, averaged over 10 runs.

The goal was to construct a biosensor that would yield a graded signal response. Due to the difficulties experienced in obtaining data, simulations were compared to that in [WWR ${ }^{+}$98], where a similar system was investigated. In that paper a graded response of the luminescent output was measured over different signal concentrations. The main parameter that affects this was found to be $\gamma_{P h z M S}$. Using the plot from [WWR ${ }^{+98}$ ], we investigated different values of $\gamma_{P h z M S}$ ranging from 170 to $500 \mu \mathrm{M}$. A graph for response over signal for each of the examined 5 values of $\gamma_{P h z M S}(10$ cells $)$ is given in Fig. 6 with standard deviation intervals of response at each value of signal. A lower value of $\gamma_{P h z M S}$ gives a more graded response, which is consistent with the results reported in [WWR ${ }^{+}$98].

For more examples and results of the stochastic analyses see [FM07]. We continue with the computationally less expensive continuous approach considering the averaged behaviour. 
Fig. 6 Diagram showing increasingly graded response to the signal for decreasing values of $\gamma_{P h z M S}(10$ cells).

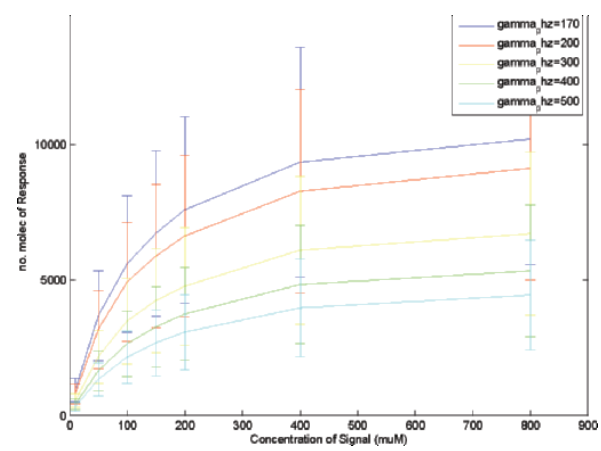

Continuous analysis In a continuous Petri net [DA05] the marking of a place is no longer an integer, but a non-negative real number, and transitions fire continuously according to the deterministic rate functions. Assigning these rate functions to all transitions, see Table 2, and reading the net in Figure 4 as a continuous Petri net, generates the ODEs as given in the equations (1) - (4). The last term in equation (1) corresponds to the pfb transition (given in grey in Figure 4).

$$
\begin{aligned}
\dot{T F} & =\alpha_{T F}-\delta_{T F} \cdot T F-\beta_{T F S} \cdot s \cdot T F+k_{d} \cdot T F S+\beta_{T F} \frac{T F S}{\gamma_{T F}+T F S} \\
T \dot{F} S & =\beta_{T F S} \cdot s \cdot T F-k_{d} \cdot T F S-\delta_{T F S} \cdot T F S \\
P h \dot{M} S S & =\beta_{P h z M S} \frac{T F S}{\gamma_{P h z M S}+T F S}-\delta_{P h z M S} \cdot P h z M S \\
P \dot{Y} O & =\alpha_{P Y O} \cdot P h z M S-\delta_{P Y O} \cdot P Y O
\end{aligned}
$$

Simulating the continuous Petri net, i.e. solving numerically the underlying system of ODEs, we get data as given in Figure 7. Here we continue the steady state analysis, comparing the kernel system against its variant in order to determine the influence of the pfb. We can see clearly that in both graphs the initial concentrations of PhzMS and PYO both start at zero. Once the reactions have begun (triggered by a pollutant) they increase until they reach a steady state. The interesting point to note is that the production of PYO and PhzMS is much higher in the system with pfb. In quantitative terms, the model with pfb gives around $30 \%$ gain. This result allows interesting insights into possible design decisions for the system under construction.

Further, we applied a variant of multi-parametric sensitivity analysis (MPSA) in order to determine those parameters which play a significant role in distinguishing the behaviour between the two models (basic version, and with pfb). Parameter $\gamma_{P h z M S}$ turned out to be the most sensitive. Thus, in order to refine our comparison 
we would first try to narrow the value range for this parameter. Such information permits prioritization of costly and time consuming wet-lab experiments.

Fig. 7 Dynamic behaviour of the continuous Petri nets allowing comparison of the two system variants. The thin curves belong to the kernel model, and the thick curves to the model with pfb.

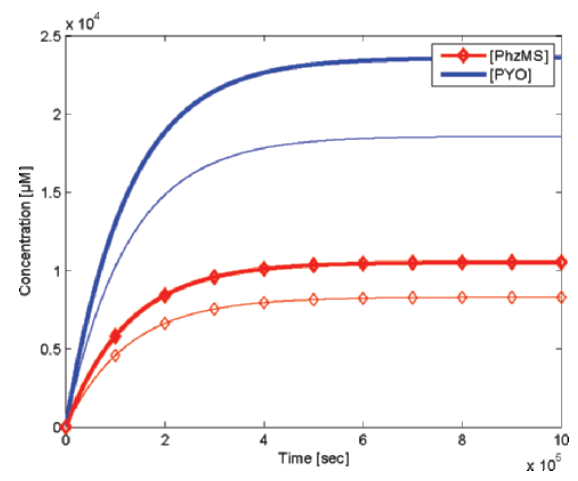

\section{Tools}

The Petri net models were built using the Snoopy [Sno, HRS08] software which is a software tool to design and animate hierarchical graphs, among others Petri nets. It supports qualitative, stochastic and continuous Petri nets, and incorporates the exact Gillespie algorithm for stochastic nets and a variety of ODE solvers for continuous nets. Snoopy provides export to various analysis tools as well as SBML import and export.

The analysis of qualitative Petri nets was performed using Charlie [Cha] which can perform analysis of structural properties, invariant analysis, reachability graph based analysis, and generate visualisations of reachability and coverability graphs. These two tools were developed at the Brandenburg University of Technology, Cottbus.

A specialised Gillespie-style "slow scale stochastic simulation algorithm" [CGP05] was coded in Matlab [MAT] in order to produce the stochastic simulations of the bio-sensor and the graphs in Figure 5 and Figure 6. Fast reactions are computed separately to the slow reactions in order to avoid the slow behaviour of a standard Gillespie-style algorithm. This approach was required due to the properties of the sensor system, where the binding and unbinding reactions of TFS are over six orders of magnitude faster than the other reactions.

The multi-parametric analysis of the model to determine those parameters which play a significant role in distinguishing the behaviour between the two models was performed using the Minicap package [Fri] implemented in Matlab and exploiting its specialised ODE solvers MATLAB [SR97]. 
The SBML version of the model was generated using the BioNessie [Bio] biochemical pathway simulation and analysis tool developed at the University of Glasgow.

\section{Summary}

The formal modelling and analysis mechanisms of Petri nets have been used in a synthetic biology project to design a completely novel type of self-powering electrochemical biosensor, called ElectrEcoBlu. The novelty lies in the fact that the response signal is an electrochemical mediator, which enables electrical current to be generated in a microbial fuel cell. The work was facilitated by a team of molecular biologists and engineers/modellers working in an integrated laboratory environment, using Petri nets as a communication means.

The 'ElectrEcoBlu' project was carried out as part of the activities of the University of Glasgow's team in the 2007 international Genetically Engineered Machines (iGEM) Synthetic Biology competition, for which they won the Environment and Sensor prize and a gold medal [iGE07].

The outcome so far is the design of a general template of a biosensor, which provably corresponds in various aspects to the desired behaviour. In the next step, the engineered cells will be constructed, i.e. they will be placed in a MFC and the electricity generated under varying conditions and pollutant concentrations will be measured. It is anticipated - supported by the model-based analyses - that the presence of the pollutant toluene would result in an enhanced reporter gene product giving rise to the electron mediator pyocyanin. This in turn is expected to increase the efficiency of electricity production resulting in a measurable electronic signal proportional to the concentration of pollutant.

The model in its three versions and more related material are available at: www.brc.dcs.gla.ac.uk/iGEM/2007.

Acknowledgements We wish to acknowledge David Leader for his valuable contribution to the determination of rate parameters, and Christine Harkness for her initial work on the continuous Petri net model. The entire Glasgow iGEM team contributed to the work of the project, including students Toby Friend, Mai-Britt Jensen, Karolis Kidykas, Martina Marbà, Linsey McLeay, Christine Merrick, Maija Paakkunianen and Scott Ramsay and staff David Forehand, Gary Gray, Margaret Jackson, Raya Khanin, Emma Travis, and Gabriela Kalna. Rachael Fulton was supported by a stipend from the Nuffield foundation. In addition we acknowledge financial support by Scottish Enterprise, the European Union NEST program, the Carnegie Trust, and the University of Glasgow as well as sponsorship from Merk and Anachem.

\section{References}

[Bio] BioNessie. A biochemical pathway simulation and analysis tool. University of Glasgow, www.bionessie.org. 
[BK02] F. Bause and P.S. Kritzinger. Stochastic Petri Nets. Vieweg, 2002.

[CGP05] Y. Cao, D.T. Gillespie, and L.R Petzold. The slow-scale stochastic simulation algorithm. Journal of Chemical Physics, 122(1):014116+, 2005.

[Cha] Charlie. A Tool for the Analysis of Place/Transition Nets. http://wwwdssz.informatik.tu-cottbus.de/software/charlie/charlie.html.

[DA05] R. David and H. Alla. Discrete, Continuous, and Hybrid Petri Nets. Springer, 2005.

[FM07] R. Fulton and M. Marba. A Stochastic Model for a General Biosensor. www.brc.dcs.gla.ac.uk/iGEM/2007, Bioinformatics Research Centre, University of Glasgow, UK, 2007.

[Fri] T. Friend. Minicap - Multi Parameter Sensitivity Analysis. Glasgow iGEm Team, http://parts.mit.edu/igem07/index.php/Glasgow/Modeling.

[GHL07] D. Gilbert, M. Heiner, and S. Lehrack. A unifying framework for modelling and analysing biochemical pathways using Petri nets. In Proc. CMSB 2007, pages 200216. LNCS/LNBI 4695, Springer, 2007.

[Gil77] D.T. Gillespie. Exact stochastic simulation of coupled chemical reactions. The Journal of Physical Chemistry, 81(25):2340-2361, 1977.

[Gla07] Glasgow University Team at iGEM - International Genetically Engineered Machine Competition. http://www.brc.dcs.gla.ac.uk/iGEM/2007/, Cited 20 Jan 2008, 2007.

[HGGH07] C. Harkness, D. Gilbert, X. Gu, and M. Heiner. The use of Petri nets in the Glasgow iGEM project: ElectrEcoBlu - a Self-powering Electrochemical Biosensor. www.brc.dcs.gla.ac.uk/iGEM/2007, Bioinformatics Research Centre, University of Glasgow, UK, 2007.

[HP06] M. Heinemann and S. Panke. Synthetic biology - putting engineering into biology. Bioinformatics, 22(22):2790-2799, 2006.

[HRS08] M. Heiner, R. Richter, and M. Schwarick. Snoopy - A Tool to Design and Animate/Simulate Graph-Based Formalisms. In Proc. PNTAP 2008, associated to SIMUTools 2008. ACM digital library, 2008.

[iGE07] iGEM - International Genetically Engineered Machine Competition, MIT. http://parts.mit.edu/igem07/, Cited 20 Jan 2008, 2007.

[MAT] MATLAB. High-level language and interactive environment. MatWorks, www.mathworks.com.

[MBC $\left.{ }^{+} 95\right]$ M. Ajmone Marsan, G. Balbo, G. Conte, S. Donatelli, and G. Franceschinis. Modelling with Generalized Stochastic Petri Nets. Wiley Series in Parallel Computing, John Wiley and Sons, 1995. 2nd Edition.

$\left[\mathrm{OAM}^{+}\right.$03] Y.Q. O’Malley, M.Y. Abdalla, M.L. McCormick, K.J. Reszka, G.M. Denning, and B.E. Britigan. Subcellular localization of Pseudomonas pyocyanin cytotoxicity in human lung epithelial cells. Am J Physiol Lung Cell Mol Physiol, 284(2):L420-430, 2003.

$\left[\mathrm{PGS}^{+}\right.$07] J.F. Parsons, B.T. Greenhagen, K. Shi, K. Calabrese, H. Robinson, and J.E. Ladner. Structural and functional analysis of the pyocyanin biosynthetic protein phzm from pseudomonas aeruginosa. Biochemistry, 46(7):1821-1828, 2007.

[Sno] Snoopy. A tool to design and animate hierarchical graphs. BTU Cottbus, CS Dep., www-dssz.informatik.tu-cottbus.de.

[SR97] L. F. Shampine and M. W. Reichelt. The MATLAB ODE Suite. SIAM Journal on Scientific Computing, 18:1-22, 1997.

[Syn08] SyntheticBiology.org. www.syntheticbiology.org, Cited 20 Jan 2008.

$\left[\mathrm{vHNM}^{+}\right.$00] J. van Helden, A. Naim, R. Mancuso, M. Eldridge, L. Wernisch, D. Gilbert, and S. J. Wodak. Representing and analysing molecular and cellular function in the computer. J Biological Chemistry, 9-10(381):921-935, 2000.

[Wil06] D.J. Wilkinson. Stochastic Modelling for System Biology. CRC Press, New York, 1st Edition, 2006.

[WWR ${ }^{+}$98] B.M. Willardson, J.F. Wilkins, T.A. Rand, J.M. Schupp, K.K. Hill, P. Keim, and P.J. Jackson. Development and Testing of a Bacterial Biosensor for Toluene-Based Environmental Contaminants. Appl Environ Microbiol., 3(64):1006-1012, 1998. 